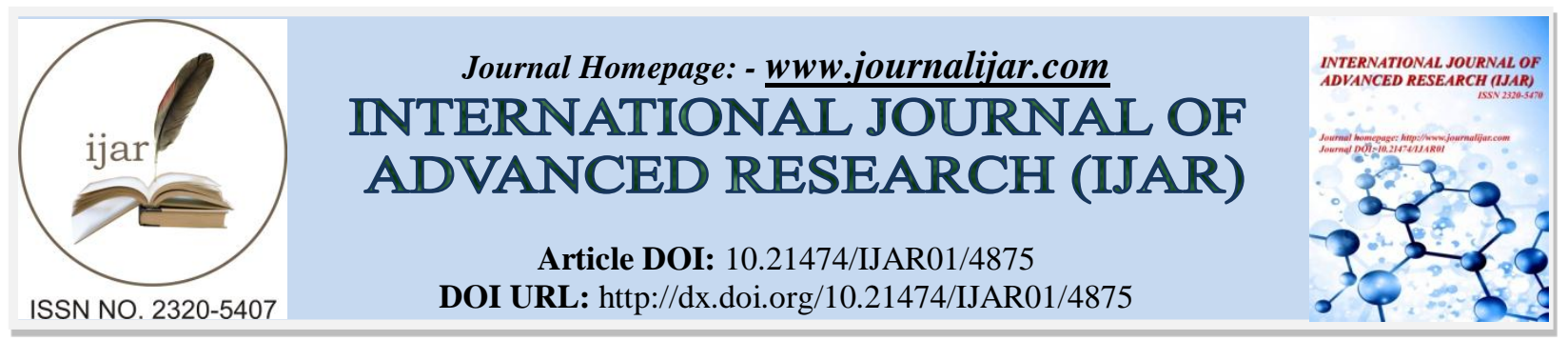

RESEARCH ARTICLE

\title{
QUALITY OF LIFE AFTER SURGERY FOR BREAST CANCER - A PILOT STUDY FROM A TERTIARY CARE HOSPITAL IN SOUTH INDIA.
}

\section{Dr Philip Umman*, Dr Joyal Jose and Dr Nigel Varghese.} Believers Church Medical College Hospital, St Thomas Nagar, Kuttapuzha, Tiruvalla 689101.

\section{Manuscript Info}

(..........................

Manuscript History

Received: 17 May 2017

Final Accepted: 19 June 2017

Published: July 2017

Key words:-

BR-23, quality of life, breast cancer, breast conservation surgery, modified radical mastectomy

\section{Abstract}

Breast cancer is the commonest cancer affecting women across all age groups. Surgery for breast cancer has evolved from radical intent and extent to wide local excision and breast conservation. Breast conservation surgery is the standard of care in the treatment of early breast cancer. However, there is very less acceptability of the same among both patients and health care professionals, especially in the Indian subcontinent. We aim to assess the quality of life in patients who underwent surgery for carcinoma breast and see the difference in quality of life, if any, between breast conservation surgery(BCS) and modified radical mastectomy (MRM) and the feasibility of the same.

Copy Right, IJAR, 2017,. All rights reserved.

\section{Introduction:-}

Breast conservation surgery (BCS) or Breast conservation treatment (BCT) is the standard of care in the treatment of early breast cancer. For selected locally advanced tumors also, BCS can be offered to the patient. However, due to various reasons, modified radical mastectomy (MRM) is the more common operation performed for early and locally advanced breast cancer. We intend to review the patients who underwent surgery for carcinoma breast, both BCS and MRM, in our institutution to study the quality of life.

\section{Materials and Methods:-}

This is a retrospective analysis of patients who underwent surgery for breast carcinoma. We collected the details of all the patients who underwent surgery for breast cancer in our hospital from October 2014 to December 2016. Ours is a tertiary care level private medical college and hospital in a tier II town of Central Travancore in Kerala. This data was obtained from a prospectively maintained log book of all surgical cases. The maximum follow up was two years and the least follow up was 3 months. All patients underwent MRM or BCS with adjuvant chemotherapy and/or hormone therapy. BCS is defined as wide local excision (WLE) with negative margins along with axillary dissection and postoperative breast irradiation.

\section{Inclusion criteria:-}

All patients who underwent surgery for breast cancer in one surgical unit were included in the study. This included both MRM and BCS. All patients included in the study had been discussed in the tumor board. Patients who underwent surgery after neo adjuvant chemotherapy were also included in the study.

\section{Exclusion criteria:-}

Patients who underwent wide local excision for suspicious cytology but did not have malignancy on frozen section or routine histopathology were excluded from the study. 
Preoperative diagnosis was obtained by fine needle aspiration cytology (FNAC) or trucut biopsy in all cases. Patients in whom the FNAC or trucut biopsy was not confirmatory but clinical examination and breast imaging suggested the possibility of breast cancer underwent intra operative frozen section followed by MRM or BCS. All the pros and cons of either modality of treatment were discussed threadbare with each patient and the decision to undergo BCS (where oncologically feasible) was taken by the patient.

We had thirty two patients meeting the inclusion criteria. Of these, sixteen patients underwent MRM and sixteen patients underwent BCS. The age ranged from 34 to 70 years. There were two male and thirty female patients. These thirty two patients were contacted through telephonic interview and asked to fill the EORTC QLQ C-30 and BR-23 questionnaire. The Malayalam language version of the form in PDF format was downloaded from the EORTC website after requisite permissions. Of the 32 patients contacted through phone, only 11 patients responded and filled the questionnaire. Five of those who responded had undergone BCS while the remaining six had undergone MRM. There was one male patient in the MRM group. Of the 10 female patients, eight were post menopausal at the time of treatment. The age range was from 34 to 68 years.

Among the six responders in the MRM group, five patients had completed the adjuvant chemotherapy also. The one male patient was undergoing chemotherapy. One patient presented with isolated opposite axilla metastasis on follow up.

Among the five responders in the BCS group, four patients had completed both adjuvant chemotherapy and breast irradiation. The fifth patient was undergoing chemotherapy.

All patients were called to the surgical outpatient clinic and given the EORTC CL Q-30 and BR-23 form to be filled up. The responses obtained were tabulated. We calculated the total number of responses according to type of response for each set of questions and analysed differences in the total number of each type of response between BCS and MRM group.

The responses in both the groups were almost on identical lines to the questionnaire. Most of the patients had completed the surgical modality of treatment and questions related to physical difficulties in the past week (Q 1-5, 616) had identical scoring patterns with most of them responding as not at all or little affected during the past week. Eight of the 11 patients cited financial difficulties following treatment (Q 28) but not much affect on their social activities (Q 27). Only one out of the eleven responders felt very much dissatisfied with their body (Q41, 42). Only three out of 11 patients answered "very much worries about their future health" response (Q43). Responses related to the operated site and ipsilateral arm movements were almost identically answered by both groups (Q47-53).

\section{Discussion:-}

From Halstedian radical mastectomy, better understanding of tumor biology and behavior, along with use of adjuvant chemotherapy and radiotherapy has led to surgery for breast carcinoma becoming less "radical". We are now in the era of breast conservation surgery, depending on the stage of the disease (Akram M, 2012).

Breast conservation surgery is the standard of care in the treatment of early breast cancer and selected cases of locally advanced breast cancer. However, there is not much acceptance of the same among patients and health care professionals. One study reported a rate of 11 to $34 \%$ for BCS. This is in contrast to the Western world, where it exceeds $70 \%$. However, when patients were given an option of breast conservation, they desired for the same (Narendra H, 2011). There is very little literature on quality of life after surgery for breast cancer in the Indian context. This study is an attempt at filling the gap. We used the EORTC CL Q-30 and BR-23 form in its Malayalam language version to record patient responses. The EORTC BR-23 form has been previously validated for use in Indian patients (Parmar V, 2005).

BCS is a less extensive surgery when compared to MRM and especially in the Western context, the cosmetic outcomes of BCS are much highlighted. However, in our patients, we found that those undergoing MRM did not feel much left out from their social activities or dissatisfied with their body. The Western concept of cosmetic outcome may not be as is applicable to our patients. However, majority of the patients in our study belong to the low or middle socio-economic class in a tier II town and its peripheries and the same may not be correct in an urban setting. Other studies have shown similar findings. In one study where patients underwent oncoplastic breast surgery to resect larger volume of tissue with wider margins, none of the patients agreed for cosmetic correction of the 
contralateral breast. The authors conclude that most of the patients were satisfied with mere preservation of the contralateral breast mound rather than symmetrical shape of the breast (Adimulam G 2014). Both categories of patients experienced financial difficulties during and as a result of treatment. There is no universal coverage of the government health insurance scheme and while surgery inflicts a onetime cost, there is recurring cost while undergoing chemotherapy and radiotherapy. Frozen section facility is required in cases with doubtful cytology to confirm the diagnosis and also comment on margins to assess adequacy of resection. This takes up operative time and precious operating theater resources and may strain the overcrowded government set up. The availability of radiotherapy facilities is also not uniform across the country. Our patients benefitted from the facility at the government medical college department. One study has suggested use of intra-operative radiotherapy to enhance the access to breast conservation surgery in resource limited developing countries. It also led to a $12 \%$ reduction in treatment related costs (Bargallo-Rocha JE, 2017).

The number of patients expressing worries about their future health was low. This is a salutary finding. It can be attributed to the good counseling of patients by a multi-disciplinary team and improved outcomes seen in patients because of early detection and proper multi-modality treatment. The better awareness of the disease process and outcomes through traditional media may also play a role.

Drawbacks of our study - The patients were not randomized to either arm of surgical treatment. Hence the two groups cannot be strictly combined. The number of people who responded to the questionnaire was low. Also the EORTC BR 23 questionnaire has questions that relate to symptoms mainly in the past week. As majority of our responders had completed all modalities of treatment, there may not be much difference in the responses to these questions. The long term outcome difference should be expected in the body appearance response. However, four out of five patients who underwent BCS and five out of six who underwent MRM responded with "not at all" or "a little" to dissatisfaction with their body status. This may again be related to the attitude and thought processes of the patients. One study found that those who underwent breast conservation treatment had a superior body image as compared to those with mastectomy. However, physical, emotional and cognitive functions were not related to the type of surgery (Parmar V, 2005).

Those who opted for MRM opt for MRM thinking that "the full disease will be removed". One study found that the main reason for refusal of BCS was the fear of recurrence in the remaining breast (Deo SS, 2001). Another study found that psychiatric state, marital adjustment and fear of cancer recurrence were similar in the two groups. They concluded that breast conservation does not in itself prevent mental sequelae after treatment for primary breast cancer (Meyer L, 1989).

There was only one recurrence in the eleven patients. However long term follow up has to be carried out. It may also be necessary to get patient responses immediately after surgery from randomized groups to know the actual difference that the two different surgical modalities bring about (Parmar V, 2005).

\section{Conclusion:-}

Both BCS and MRM are equally good modalities of treatment for Indian patients for early breast cancer and selected cases of locally advanced breast cancer. However, larger studies are needed to arrive at definitive conclusions. Longitudinal studies of the same patient will also help in identifying how the different modalities of treatment in breast cancer affect responses. 


\section{References:-}

1. Adimulam G, Challa VR, Dhar A,Chumber S, Seenu V,Srivastava A.. Assessment of cosmetic outcome of oncoplastic breast conservation surgery in women with early breast cancer: a prospective cohort study. Indian J Cancer. 2014 Jan-Mar;51(1):58-62

2. Akram M, Siddiqui SA. Breast cancer management: past, present and evolving. Indian J Cancer. 2012 JulSep;49(3):277-82.

3. Bargallo-Rocha JE, Soto-Perez-de-Celis E, Picó-Guzmán FJ, Quintero-Rodríguez CE et al. The impact of the use of intraoperative radiotherapy on costs, travel time and distance for women with breast cancer in the Mexico City Metropolitan Area. J Surg Oncol. 2017 Jun 12.

4. Deo SS, Mohanti BK, Shukla NK, Chawla S, Raina V, Julka PK, Rath GK. Attitudes and treatment outcome of breast conservation therapy for stage I \& II breast cancer using peroperative iridium-192 implant boost to the tumour bed. Australas Radiol. 2001 Feb;45(1):35-8.

5. Meyer L, Aspegren K. Long-term psychological sequelae of mastectomy and breast conserving treatment for breast cancer. Acta Oncol.1989;28(1):13-8.

6. Narendra H, Ray S. Breast conserving surgery for breast cancer: single institutional experience from Southern India. Indian J Cancer. 2011 Oct-Dec;48(4):415-22.

7. Parmar V, Badwe RA, Hawaldar R, Rayabhattanavar S, Varghese A, Sharma R, Mittra I. Validation of EORTC quality-of-life questionnaire in Indian women with operable breast cancer. Natl Med J India. 2005 Jul-Aug; 18(4):172-7. 